\title{
АЛГОРИТМ ФОРМИРОВАНИЯ ПЛАНОВ ДЕЙСТВИЙ ПО ЛИКВИДАЦИИ ЧРЕЗВЫЧАЙНЫХ СИТУАЦИЙ
}

\section{THE STRUCTURE OF THE ALGORITHM OF FORMATION OF PLANS FOR COMBAT OPERATIONS DURING RESCUE OPERATIONS IN EMERGENCIES}

\section{N. Rozhkova}

Summary. The formation of combat action plans during emergency rescue operations (ASR) is carried out on the basis of an algorithm that combines ontological modeling of the subject area with an algorithm for generating combat action plans.

Keywords: ontological modeling, rescue operations, plan generation algorithm.

\section{Вемение}

$\mathbf{H}$ аибольшее напряжение в управлении деятельностью по организации ликвидации чрезвычайных ситуаций (ЧС) создается в первые часы и даже минуты с момента их возникновения. Гарантия своевременности и полноты выполнения всех необходимых мероприятий достигаются заблаговременной разработкой типовых алгоритмов действий руководителей ликвидацией чрезвычайных ситуаций (РЛчС) и привлекаемых к ликвидации ЧС подразделений Единой государственной системы предупреждения и ликвидации чрезвычайных ситуаций (РСЧС). Однако, при возникновении чрезвычайных ситуаций в условиях ограниченного времени и быстро меняющейся оперативно-тактической обстановки, складывающейся на месте ЧС, требуется привязка раннее разработанных типовых алгоритмов действий к конкретным объектам, местности, времени, имеющимся в наличии сил и средств РСЧС.

\section{Принчип формирования алгоритма}

Аварийно-спасательные и другие неотложные работы (АСДНР), проводимые на месте ЧС, характеризуются большим объемом и ограниченностью времени на их проведение, сложностью оперативно-тактической обстановки и предельным напряжением сил всего привлечённого личного состава РСЧС. Планирование боевых действий при проведении АСДНР осуществляется на основе управленческих решений руководителя лик-
Рожкова Наталия Владимировна

Адъюнкт, Академия Государственной противопожарной службы МЧС России nvr9@mail.ru

Аннотация. Статья посвящена формированию планов боевых действий при проведении аварийно-спасательных и других неотложных работ (АСДНР), которое осуществляется на основе алгоритма, объединяющего онтологическое моделирование предметной области с алгоритмом генерации планов боевых действий.

Ключевые слова: онтологические моделирование, аварийно-спасательные работы, алгоритм генерации планов.

видацией чрезвычайных ситуаций (РЛЧС). При этом разрабатывается тактический план боевых действий при проведении АСДНР. На данном этапе производится дополнение онтологической модели данной предметной области (ПРО) с алгоритмом генерации проектов планов боевых действий.

При этом учитываются:

- характеристика оперативной обстановки на текущий момент времени, то есть значения атрибутов объектов ПРО, обобщений, процессы, события и действия оперативных групп (ОГ) территориальных подразделений МЧС России;

- множество целей, которые могут быть поставлены перед ОГ в данной обстановке;

- определение порядка приоритета достижения целей.

В районе возникновения ЧС оперативная обстановка динамически изменяется. Поэтому разработанные планы могут существенно устаревать. Для их быстрого обновления, необходимо постоянно обновлять рабочую память планировщика системы поддержки принятия решений. Обновление рабочей памяти должно занимать минимальное время. Для этого необходим алгоритм, позволяющий автоматически или с участием РЛЧС выполнять функцию прогнозирования состояния оперативной обстановки в районе ЧС на текущий момент времени. Логическая связь, объединяющая онтологическую модель с алгоритмом генерации управляющих решений, приведена на рисунке 1. 


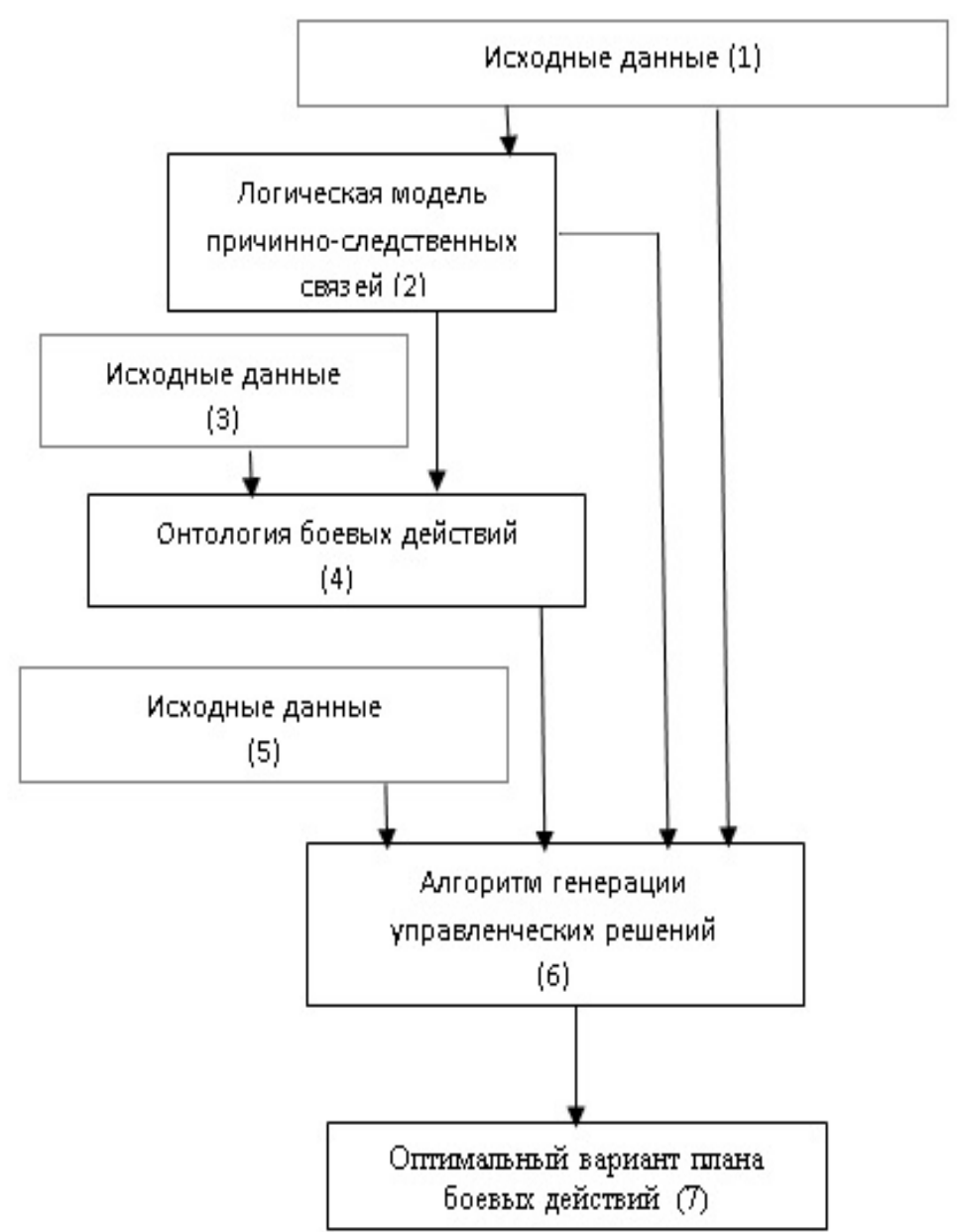

Рис. 1. Последовательность объединения онтологической модели с алгоритмом генерации управляющих планов боевых действий

Из полученных оперативных данных определяются признаки, осуществляется анализ происходящих событий, процессов, проводится их обобщение и вырабатываются действия, которые в качестве исходных данных (1) вводятся в (2). К результату моделирования в качестве исходных данных добавляются значения обобщений (3). Полученные обобщённые данные обрабатываются в модели онтологии боевых действий (4). Результатом работы модели определяются цели и их приоритеты. К полученным результатам моделирования в качестве исходных данных добавляются цели и ограничения (5) которые передаются в алгоритм генерации управляющих решений (6). Дополнительно на 6 передаются данные, сформированные в (1) и в (2). В результате работы (6) формируется оптимальный вариант плана боевых действий (7) с учетом действий участников, проводящих аварийно-спасательные работы и другие действия.

В предлагаемой онтологической модели причинно-следственных связей (ПСС) прецеденты представлены в виде вариантов ПСС. При этом алгоритм в каждый момент времени использует информационные данные, получаемые в результате выбора различных вариантов, которые описывают вероятные сценарии развития оперативной обстановки.

Таким образом, сформирован алгоритм иерархического сетевого формирования проектов планов боевых действий (ИСП БД) при проведении АСДНР. В нём использованные аккумулированные в базе знаний прецеденты основываются на алгоритме HTN — планирования (иерархического сетевого планирования), который предложен Erol K., Handler J., Nau D. S. [1, 2, 3].

Основные отличие предлагаемого алгоритма ИСП БД от классического HTN-алгоритма заключаются в следующем:

1. Предложен механизм генерации тактических планов боевых действий при проведении АС ДНР. В нём предусмотрено параллельное выполнение нескольких боевых задач.

2. Предусмотрены временные ограничения на выполнение боевых задач. 


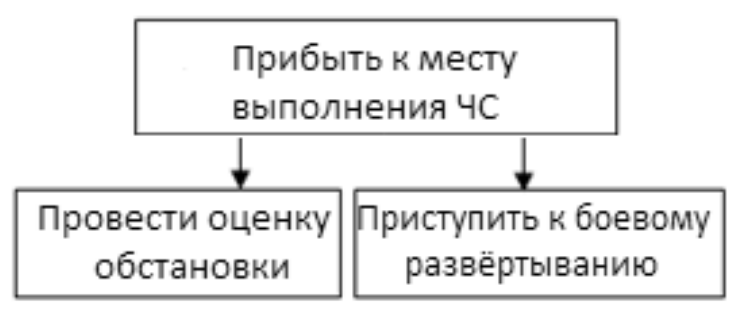

Рис. 2. Пример формирования сети задач

3. Рассмотрен новый тип задач - обеспечивающая задача.

4. Изменен принцип формирования плана. Если в HTN осуществляется последовательный перебор всех элементарных задач и автоматически определяется порядок их выполнения, удовлетворяющий всем введенным ограничениям и при этом $[1,2,3]$ предполагается, что все возможные примитивные задачи и методы получения составных задач уже существуют в базе данных, а сгенерированный план всегда есть упорядоченная совокупность только примитивных задач, то в алгоритме ИСП БД эти требования исключены, т.е. база знаний может быть не полной и план может состоять как из элементарных, так и составных задач.

5. Применённый метод решения боевой задачи производится не простым последовательным перебором имеющихся в базе данных методов, а используется критерий близости с прецедентом в базе знаний.

С учетом рассмотренных отличий ИСП БД и был дополнен синтаксис языка и адаптирована семантика, лежащие в основе алгоритма HTN — планирования.

\section{Особенности провеАения иерархического сетевого п^анировании боевых Аействий}

Исходные условия, определяющие автоматическое формирование тактических планов боевых действии при проведении АСДНР предусматривает множество целей, таких как - спасение людей, ликвидация очага пожара, защита объектов, эвакуация имущества. В это же время накладываются и ограничения. Они устанавливают порядок их достижения. Ограничения формируются на основе правил, определенных онтологией боевых действий и значений обобщений, которые введены в результате функционирования модели ПСС или введенных РЛЧС.

При иерархическом сетевом планировании боевых действий (ИСП БД) каждое состояние среды представля- ется множеством элементарных задач, имеющих в этом состоянии значение «истина». Действия соответствуют переходам состояний, т.е. каждое действие - это частичное отображение множества состояний на множество состояний. В рассматриваемой ИСП БД происходит поиск методов реализации целей, которые соответствуют сетям задач (рисунок 2).

Сеть задач - это некоторая совокупность задач, которые необходимо выполнить, ограничений, которые учитывают порядке их выполнения, способов инициации переменных, и логических выражений, которые равны перед выполнением или после выполнении задачи.

Планирование происходит через декомпозицию задач и разрешение конфликтов.

Если сеть задач, содержит только элементарные задачи, то она называется элементарной сетью задач (ЭСЗ).

В общем случае сеть задач может содержать неэлементарные задачи (рисунок 2). Неэлементарные задачи представляют собой действия, которые предполагают выполнение множества других задач. Они не могут выполняться напрямую. В качестве примера решения неэлементарной задачи рассматривается алгоритм ИСП БД.

Пусть имеются задачи планирования боевых действий в плане Р, которые содержат как элементарные задачи, так и неэлементарные задачи. Вначале проводится проверка на наличие только элементарных задач. Следующим шагом рассматривается первая неэлементарная задача а, для которой выбирается метод-расширение и расширением заменяется задача а. Из процедуры расширения конфликтов выбирается тот способ, который ближе всего соответствует решению задачи а. Далее осуществляется возврат для выбора и анализу следующих неэлементарных задач. Исходя из этого, алгоритм решения неэлементарных задач выглядит следующим образом:

Шаг 1. Если план Р содержит только элементарные задачи, то разрешить конфликты в плане Р и возвратить результат. Если конфликт не может быть разрешен, возвратить неудачу. 


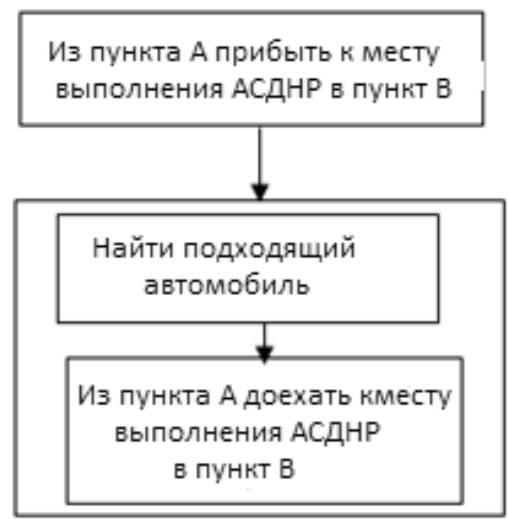

Рис. 3. Пример метода решения задачи «Доставить спасателей из пункта А в пункт В»

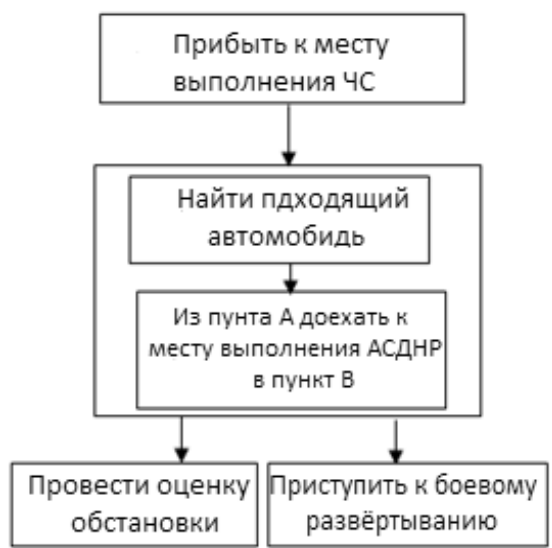

Рис. 4. Пример декомпозиции задачи «Прибыть к месту проведения ЧС»

Шаг 2. Выбрать неэлементарную задачу а в плане Р.

Шаг 3. Выбрать метод-расширение для задачи $\alpha$.

Шаг 4. Заменить задачу а расширением.

Шаг 5. Использовать процедуры разрешения конфликтов в плане P.

Шаг 6. Применить один из способов, предложенных на шаге 6.

Шаг 7. Возврат к шагу 2.

Способы выполнения представленных в плане Р неэлементарных задач используют конструкции, называемые методами.

Метод - это семантическая конструкция вида $(\alpha, \mathrm{d})$. В ней $\alpha$ определяет неэлементарную задачу, a d определяет сеть задач. При этом считается, что для выполнения задачи $\alpha$ необходимо, без нарушения ограничений в d, выполнение всех задач в сети задач d.

Работает алгоритм ИСП БД итеративно. При этом декомпозируются задачи и разрешая конфликты до тех пор, пока не будет найден бесконфликтный план, состоящий только из элементарных задач.
Расширение каждой неэлементарной задачи (шаги 2-4 алгоритма) осуществляется путем нахождения метода, посредством которого задача может быть выполнена и заменой неэлементарной задачи сетью задач, которые соответствуют этому методу.

Рассмотрим задачу (рисунок 2) прибытия спасательных подразделений к месту выполнения АСДНР. Эта задача может быть выполнена различными способами: поездом, на автомобилях или вертолетом. Например, если доставка подразделений осуществляется с использованием автомобиля, которая включает решение целого комплекса подзадач: нахождение подходящего автомобиля, заправка (дозаправка) его топливом, согласование маршрута следования и т.д. Однако, такой выбор доставки сил и средств возможен только при выполнении определенных условий. Это наличие подъездных дорог, целостности мостов и наличие переправ и т.д. Декомпозиция задачи (рисунок 2) показана на рисунке 3.

Задача «Прибыть к месту выполнения ЧС» (рисунок 2) может быть расширена, используя метод, представлен- 
ный на рисунке 3, произведя сеть задач, показанный на рисунке 4.

Сеть задач, произведенная на шаге 5, может содержать конфликты, являющиеся следствием взаимодействий среди задач. Например, если мы потратим достаточно много времени на поиск транспортного средства (например, автомобиля), то мы можем потерять шансы спасти людей или потушить пожар. Функции нахождения и разрешения таких конфликтов, например, введением дополнительных ограничений, выполняются соответствующими процедурами, называемыми критикой.

Критика впервые была введена в системе NOAH [1, 2], для идентификации и управления различного вида взаимодействиями в различных сетях задач. Эта функция от- ражена в 6 и 7 шаге: после каждой редукции, множество критических правил применяется для распознавания и разрешения взаимодействий между текущей и любыми другими редукциями. Таким образом, критика обеспечивает общий механизм для раннего обнаружения взаимодействий.

\section{Зак^ючение}

В статье рассмотрены вопросы формирования алгоритма принятия управленческих решений по выполнению боевой задачи при проведении АСДНР. Рассмотрен вариант декомпозицию неэлементарной задачи в элементарную. При этом декомпозируются задачи и, разрешая конфликты до тех пор, пока не будет найден бесконфликтный план, состоящий только из элементарных задач.

\section{ЛИТЕРАТУРА}

1. Приказ МЧС России от 16 октября 2017 года № 444 «06 утверждении Боевого устава подразделений пожарной охраны, определяющего порядок организации тушения пожаров и проведения аварийно-спасательных работ».

2. Приказ МЧС России от 25 октября 2017 года № 467 «06 утверждении Положения о пожарно-спасательных гарнизонах».

3. Указание МЧС России от 3 сентября 2013 года № 2-4-87-25 «Методические рекомендации по внедрению и организации функционирования программно-аппаратного комплекса автоматизированной геоинформационной системы поддержки принятия решений и оперативного управления подразделениями гарнизона пожарной охраны при предупреждении и ликвидации чрезвычайных ситуаций, тушении пожаров на территории субъекта Российской Федерации (ПАК «АРГО»)».

4. Брушлинский Н. Н., Микеев А. К., Бозуков Г. С. и др. Совершенствование организации и управления пожарной охраной. - М.: Стройиздат, 1986.- 152 с.

5. Мешалкин Е. А., Олейников В. Т., Абрамов А. П. Структура программно - аппаратного комплекса поддержки принятия решений для руководителя тушения пожара // Сб. тр.ч.2 «Крупные пожары предупреждение и тушение».— М.: ВНИИПО МВД России, 2001. — с. 520-524.

6. Erol K., Handler J., Nau D. S. HTN Planning: Complexity and Expressivity In Proceedings of AAAI-94, Seattle, July 1994, pp. 1123-1128. http://www.cs.umd.edu/ users/kutluhan/Papers/AAAl-94.ps

7. Erol K., Handler J., Nau D. S. Semantics for Hierarchical Task-Network Planning. Technical report CS-TR-3239, UMIACS-TR-94-31, ISR-TR-95-9, Computer Science Dept., University of Maryland. March 1994. http://citeseer.nj.nec.com/erol94semantics.html

8. Takeda H., Takaai M., and Nishida T. Collaborative development and Use of Ontologies for Design, Proceedings of the Tenth International IFIP WG 5.2/5.3 Conference PROLAMAT 98, September 9-10-11, 12, Trento, Italy. http://ai-www.aist-nara.ac.jp/doc/papers/takeda/pdf/prolamat98final.pdf 